\title{
Effects of bead-bead interactions on the static and dynamical properties of model polymer solutions
}

\author{
C. Xiao and D. M. Heyes \\ Department of Chemistry, School of Physical Sciences, University of Surrey, Guildford GU2 5XH, United Kingdom
}

(Received 22 February 1999)

\begin{abstract}
The effects of segment-segment interactions on the static and dynamical properties of model polymer solutions are examined by Brownian dynamics simulations in the free-draining limit over a wide concentration range. A bead-and-spring model is used to describe the polymer chains at a coarse-grained level, in which segment-segment interactions are represented by a bead-bead pair potential with a Gaussian analytic form, $\beta u_{\mathrm{ev}}(r)=A \exp \left(-r^{2} / 2 \sigma^{2}\right)$, where $\beta=1 / k_{B} T$ and $A$ and $\sigma$ are characteristic energy and distance scales, respectively. The chain dimensions, self-diffusion coefficient, and viscosity of the systems are studied as functions of number density of beads of the system, $\rho$, at given excluded-volume potential parameters, $A$ and $\sigma$. Our results show that in the limit of infinite dilution even for short chains $(N \sim 10)$ there is statistically significant scaling behavior in the static and dynamical properties. For a system with given values of $A$ and $\sigma$ the change in polymer coil size shows a realistic trend as the concentration of the system increases. In the dilute and concentrated regions the coil size decreases as a result of increasing interchain repulsions, while in the highly concentrated region the coil size increases again, showing a return to Rouse-like behavior because the intrapolymer and interpolymer segment-segment interactions become effectively indistinguishable for an arbitrary bead and to a large extent are "balanced out." In the limit of infinite dilution, the self-diffusion coefficient of the center of mass, $D_{\mathrm{cm}}$, depends on $N$ only and not on the potential parameter $A$, while in contrast the specific viscosity $\eta_{\mathrm{sp}}$ depends on both $N$ and $A$. As the concentration increases $D_{\mathrm{cm}}$ decreases and $\eta_{\mathrm{sp}}$ increases consistent with the behavior of real polymers. When the system becomes highly concentrated, however, both $D_{\mathrm{cm}}$ and $\eta_{\mathrm{sp}}$ unrealistically return to the Rouse limit. This suggests that from the concentrated region upward in concentration, the entanglement or the topological constraints caused by the physical connectivity of the chains significantly influence their dynamical behavior. The mean-field segment-segment interactions or excluded-volume effects incorporated in the current coarse-grained bead-spring approach cannot capture this entanglement effect, and therefore give rise to unrealistic dynamical behavior in the concentrated regime.
\end{abstract}

[S1063-651X(99)05911-5]

PACS number(s): $61.25 . \mathrm{Hq}$

\section{INTRODUCTION}

Polymer chains in solution exhibit many interesting structural and rheological features in different concentration regions. Experiments show that despite the complexity of polymers and a wide variation in atom-level detail, their macroscopic/coarse-grained behavior follows universal scaling laws [1-6]. This is the case even for biopolymers that have more specific interactions between the backbone and side chains of the molecule than synthetic polymers [7-9]. Based on the observation that the macroscopic behavior of polymer molecules is dominated by a characteristic length scale of the molecule, various scaling theories have been proposed that give coarse-grained predictions for structural and dynamical properties in different concentration regions [10-13], and which have been confirmed to a large extent by both experiment [14-17] and computer simulation [18-25]. While the properties of polymer chains at infinite dilution and in the melt state have often been simulated, there is a need for similar studies in the intermediate region for solutions, where the correlation length of density fluctuations is comparable to the coil size of the polymers, and also at higher concentrations approaching the melt limit. In this study we have carried out Brownian dynamics simulations for a system of bead-and-spring chains in this concentration range as well as at infinite dilution.

The physical properties of a polymer solution are determined by at least four features of the polymer molecules: (a) the connectivity of the backbone, (b) the segment-segment interactions, (c) the hydrodynamic interactions (HI) between polymer segments and solvent molecules, and (d) the uncrossability of the chains, which cause entanglement at the molecular level. While it could reasonably be argued that many-body hydodynamic interactions can be neglected at high concentrations and entanglement in dilute solution, the connectivity of the backbone and the segment-segment interactions are effective throughout the whole concentration region. In this study we have focused on the connectivity and the segment-segment interactions to investigate their contribution to both structural and dynamical properties of polymer chains in solution. Also, the absence of true molecularlevel topological entanglement in our model allows us to draw some conclusions as to its possible consequences.

The simplest model to describe the connectivity of a polymer chain is the Rouse model [26], in which a polymer chain is described in terms of a set of $N$ beads linked sequentially by $N-1$ linear springs with a potential $\beta u(l)=\frac{3}{2} l^{2}$, where $\beta=1 / k_{B} T$ and $l$ is the spring bond vector between two linked beads. Although the spring potentials enable correlated movements of the beads along the chain, the beads are not physically linked and the chains can cross each other freely. 
Excluded volume (EV) has been introduced in the form of bead-bead interactions to prevent two beads from occupying the same space [27-29]. However, the way the EV potential is defined means that it reflects both the chain chemistry and the solvent quality, $\mathbf{F}_{\mathrm{ev}}=\mathbf{F}_{0}+\mathbf{F}_{m}$, where $\mathbf{F}_{0}$ is the repulsive force between any two unlinked beads when the chains are in vacuum and $\mathbf{F}_{m}$ is the solvent mediation force caused by the bead-solvent interactions when the chains are immersed in a solvent. Obviously, $\mathbf{F}_{0}$ depends on the molecular details of the chain. The stronger the $\mathbf{F}_{0}$, the stiffer the chain. $\mathbf{F}_{m}$ depends on the affinity of the chains towards the solvent. If the bead-solvent interactions tend to pull the beads apart, $\mathbf{F}_{m}$ is repulsive; otherwise it is attractive. The total force, the socalled "excluded-volume force," determines the quality of the solvent for a given polymer. In a good solvent, $\mathbf{F}_{\mathrm{ev}}>0$ and the repulsions keep the beads further apart than for the Rouse chains. In a bad solvent, $\mathbf{F}_{\mathrm{ev}}<0$ and the beads are forced together, causing the chains to tend to collapse. In a $\theta$ solvent, $\mathbf{F}_{\mathrm{ev}}=0$, there are no net forces between unlinked beads, and the chains reduce to Rouse chains. The consequences of the total force $\mathbf{F}_{\mathrm{ev}}$ is called the excluded-volume (EV) effect.

The EV force plays an important role in polymer solutions and this can be represented by a simple pair potential between the beads. From a theoretical point of view, the effects of the analytical form of the EV potential on the structural and dynamical properties of these systems are largely unknown. In this work we propose a model EV potential and have carried out Brownian dynamics (BD) simulations on these model polymer solutions to examine its effect on the behavior of polymer chains in solution. We explore the statistical distribution of the chain segments, the diffusion coefficient of the center of mass, and the viscosity. We have investigated a wide concentration range spanning either side of the chain overlap concentration, $\rho^{*}$, which we will define precisely below.

\section{THEORETICAL BACKGROUND}

Consider a solution of $N_{\mathrm{ch}}$ bead-spring chains in a volume $V$ with spring potential $u_{\mathrm{sp}}(\Delta \mathbf{I})$ and EV potential $u_{\mathrm{ev}}(\mathbf{r})$. Let $\boldsymbol{l}$ be the spring bond vector between any two linked beads, $\mathbf{r}$ the distance between any two unlinked beads, and $\Delta \boldsymbol{l}=\boldsymbol{l}$ $-\boldsymbol{l}_{0}$, with the subscript 0 indicating the natural state of the spring. The number density of the chains in the system is $\rho_{\mathrm{ch}} \equiv N_{\mathrm{ch}} / V$ and the number density of beads is $\rho$ $\equiv N N_{\text {ch }} / V$, where $N$ is the number of beads per chain.

In a $\theta$ solvent, if the many-body hydrodynamic interactions (MHI) are neglected, the chains behave like Rouse chains. In a good solvent, however, the EV interactions are influential. At extreme dilution, the chains are essentially isolated, and therefore intrachain EV effects dominate, although chains do interact occasionally because of their open structure. The intrachain EV effect pushes the beads away from each other, causing the chain to swell. The chains form larger coils than in a $\theta$ solvent. As the solution becomes more concentrated, the chains increasingly interact with and interpenetrate each other so that the interactions between the beads from different chains become more important. For any bead on a given chain it increasingly experiences the effects of beads from other chains. As a result, the intrachain EV effect becomes less important and the chains shrink. When the system becomes highly concentrated, the system is more homogeneous and the density fluctuations decrease in magnitude. On average, the forces on each bead tend to balance out because of the high level of occupation of the first coordination shell. The beads begin to lose awareness of the connectivity of the chains since, because the beads are packed closely together, it is difficult for any particular bead to distinguish which chains other beads belong to. The net force on a given bead exerted by the surrounding beads tends to zero and the chains become, in the limit of the melt, Rouselike chains again.

We will term the region where intrachain repulsions dominate as "dilute," the region where interchain repulsions dominate as "concentrated,' and the region where intrachain and interchain repulsions are undistinguishable as "'highly concentrated.' In the beginning of the concentrated range, there is a region where the chain density $\rho_{\mathrm{ch}}$ is still very low despite the high bead density $\rho$. This region is called "semidilute." The longer the chains, the more extended the semidilute region.

We can define an overlap density,

$$
\rho^{*} \equiv \frac{N}{\frac{4}{3} \pi R_{\mathrm{gyr}}^{3}}
$$

where $R_{\mathrm{gyr}}$ is the root-mean-square radius of gyration of the polymer chains at $\rho^{*}$ (which is quite close to the infinite dilution value). This is the number density of beads when chains start to overlap, providing a geometrical prescription for the transition from dilute region to concentrated region. The open structure of the chain molecules ensures that once the system leaves the limit of infinite dilution, the interchain interactions gradually increase so that $\rho^{*}$ does not represent a phase transition.

There are various theories attempting to explain the behavior of polymer solutions in these different concentration ranges. While theories for infinite dilute systems and the melt are very successful, more needs to be done for the concentrated region. We summarize here some established scaling laws so that we can conveniently compare our simulation results with them [30]. We denote the root-mean-square bond length $\left(\left\langle l^{2}\right\rangle\right)^{1 / 2}$ as $b$, and the position vector of bead $j$ as $\mathbf{R}_{j}$. In the limit of infinite dilution we have the following relations. The mean-square distance between any two beads $m$ and $n$ on the same chain, $S_{m n}^{2}$, is

$$
S_{m n}^{2} \equiv\left\langle\left(\mathbf{R}_{n}-\mathbf{R}_{m}\right)^{2}\right\rangle \propto|m-n|^{2} \nu b^{2},
$$

where $\nu$ is an exponent that depends on the solvent quality. The mean-square radius of gyration, $R_{\mathrm{gyr}}^{2}$, is

$$
R_{\mathrm{gyr}}^{2} \equiv \frac{1}{N} \sum_{j=1}^{N}\left\langle\left(\mathbf{R}_{j}-\mathbf{R}_{\mathrm{cm}}\right)^{2}\right\rangle \propto N^{2 \nu} b^{2}
$$

where $\mathbf{R}_{\mathrm{cm}}$ is the position of the center of mass of the chain defined by

$$
\mathbf{R}_{\mathrm{cm}} \equiv \frac{1}{N} \sum_{j=1}^{N} \mathbf{R}_{j}
$$


Turning now to dynamical properties, the self-diffusion coefficient for the center of mass of the chain, $D_{\mathrm{cm}}$, is

$$
D_{\mathrm{cm}} \equiv \lim _{t \rightarrow 0} \frac{1}{6 t}\left\langle\left[\mathbf{R}_{\mathrm{cm}}(t)-\mathbf{R}_{\mathrm{cm}}(0)\right]^{2}\right\rangle \propto N^{-\nu_{D}},
$$

where $\nu_{D}$ is a characteristic exponent for $D_{\mathrm{cm}}$. Let $\eta$ be the viscosity of the solution and $\eta_{s}$ that of the pure solvent. For the intrinsic viscosity, $[\eta]$, we have

$$
[\eta] \equiv \lim _{\rho \rightarrow 0} \frac{\eta-\eta_{s}}{\eta_{s} \rho} \propto N^{\nu} \eta b^{2},
$$

where $\nu_{\eta}$ is the associated scaling exponent. The values for these exponents in some well defined limits are as follows:

$$
\begin{gathered}
\nu=\left\{\begin{array}{l}
0.5, \quad \text { Rouse chains }(\theta \text { solvent }) \\
0.588, \quad \text { self-avoiding chains (good solvent)}
\end{array}\right. \\
\nu_{D}= \begin{cases}1, & \text { Rouse model } \\
\nu, & \text { Zimm model }\end{cases} \\
\nu_{\eta}= \begin{cases}1, & \text { Rouse model } \\
3 \nu-1, & \text { Zimm model }\end{cases}
\end{gathered}
$$

The above results are calculated from self-avoiding chains, for which the EV potential is taken as a delta function. In a $\theta$ solvent, self-avoiding chains become Rouse chains. If we take into account many-body hydrodynamic interactions in self-avoiding chains, we obtain the Zimm model [31]. It is important to include MHI effects to obtain realistic values for the dynamic scaling exponents $\nu_{D}$ and $\nu_{\eta}$. If we take the value 0.6 for $\nu$, the Zimm model values agree well with those obtained for real polymers, i.e., $\nu_{D}$ $=0.5, \nu_{\eta}=0.5$ in a $\theta$ solvent and $\nu_{D}=0.6, \nu_{\eta}=0.8$ in a good solvent. However, the values of the static scaling exponent $\nu$ agree well with real polymers under different solvent conditions without the need for MHI in the model.

In concentrated solutions, the situation becomes more complicated. Since the concentration fluctuations are still large and the correlations between segments are strong, a polymer solution in the concentrated region assumes critical behavior. The influence of the number density of beads $\rho$ on the static and dynamic properties of the polymer solution can be analyzed using scaling arguments that in a good solvent give the following predictions for the density dependence of the static and dynamical properties $[13,30]$ :

$$
\begin{aligned}
\alpha_{\mathrm{gyr}} & \equiv \frac{R_{\mathrm{gyr}}(\rho)}{R_{\mathrm{gyr}}(0)} \propto\left(\frac{\rho}{\rho^{*}}\right)^{\kappa}, \\
\alpha_{\mathrm{cm}} & \equiv \frac{D_{\mathrm{cm}}(\rho)}{D_{\mathrm{cm}}(0)} \propto\left(\frac{\rho}{\rho^{*}}\right)^{\kappa_{D}}, \\
\eta_{r} & \equiv \frac{\eta(\rho)}{\eta_{s}} \propto\left(\frac{\rho}{\rho^{*}}\right)^{\kappa_{\eta}},
\end{aligned}
$$

where $\kappa=(1-2 \nu) / 2(3 \nu-1), \quad \kappa_{D}=(2-\nu) /(3 \nu-1)$, and $\kappa_{\eta}=3 /(3 \nu-1)$. For $\nu=0.6$ we have $\kappa=-0.125, \kappa_{D}$ $=-1.75$, and $\kappa_{\eta}=3.75$, respectively.

\section{SIMULATION AND MODEL DETAILS}

Our model system consists of $N_{\mathrm{ch}}$ linear spring-and-bead chains each with $N$ identical beads. The position vector for a bead $j$ is $\mathbf{R}_{j}$, the spring bond vector between any two linked beads is $\boldsymbol{l}_{k} \equiv \mathbf{R}_{k+1}-\mathbf{R}_{k}$, and the distance between any two unlinked beads is $\mathbf{r}_{m n} \equiv \mathbf{R}_{n}-\mathbf{R}_{m}$. We assume that the Brownian forces acting on the beads are uncorrelated and therefore the long-time dynamics of the beads satisfy the position Langevin equation [32,33]. Neglecting many-body hydrodynamic interactions, we have for bead $j$ a bead position update scheme in a form convenient for simulation [34],

$$
\mathbf{R}_{j}(t+\Delta t)=\mathbf{R}_{j}(t)+\mathbf{F}_{j}(t) \frac{\Delta t}{\zeta}+\Delta \mathbf{R}_{j}^{G}(t),
$$

where $\zeta$ is the friction coefficient of the bead characterizing the bead-solvent interaction, and $\Delta \mathbf{R}_{j}^{G}$ is a random Brownian displacement acting on bead $j$ taken from a Gaussian random number generator. The thermodynamic or excluded-volume force $\mathbf{F}_{j}$ on a bead is determined by the potential field of the system, which here has two parts - the sum of the spring forces from the two beads linked to it and the sum of the EV forces from all the unlinked beads around it.

Two analytic forms of spring potential were used in our simulations. For the class of systems denoted by the key, $F 0$, we used a linear spring with zero natural length i.e., $l_{0}$ $=0.0$ so that $\Delta l=l$ and the interaction potential was,

$$
\beta u(l)=\frac{H}{2} l^{2}
$$

where $H$ is a characteristic energy. This is the spring potential used in the Rouse model. For the class of systems denoted by $F 1$, we use a finitely extendible nonlinear elastic (FENE) spring model [35] that has a nonzero equilibrium natural length $l_{0}$ and a maximum extension $\Delta l_{\max }$,

$$
\beta u(\Delta l)=-\frac{H}{2} \Delta l_{\max }^{2} \ln \left(1-\frac{\Delta l^{2}}{\Delta l_{\max }^{2}}\right),
$$

where $\Delta l=\left|\boldsymbol{l}-\boldsymbol{l}_{0}\right|$. We used the values $H=3.0$ and $\Delta l_{\max }$ $=3.0 l_{0}$ in this study. The FENE spring potential for $F 1$ systems guarantees that the springs do not get overstretched at high concentrations. In the limit $\Delta l \rightarrow 0$ the FENE potential reduces to a linear harmonic spring interaction potential, $\beta u(\Delta l)=\frac{3}{2} \Delta l^{2}$.

Turning now to the nonbonded interaction, the EV potential we used had a Gaussian analytical form,

$$
\beta u_{\mathrm{ev}}(r)=\left\{\begin{array}{l}
A e^{-r^{2} / 2 \sigma^{2}}, \quad r<4 \sigma \\
0, \quad r \geqslant 4 \sigma,
\end{array}\right.
$$

where $\sigma$ sets the range of the potential and $A$ the energy scale or strength of the interaction. Despite this seemingly arbitrary choice, it is a plausible potential form, since it allows for progressively more difficult interpenetration of the beads as the values of $A$ and $\sigma$ increase. The barrier height at $r$ $=0$ is finite, however. This is a realistic feature, because each bead represents a substantial section of a polymer chain. The dimensionless exponential prefactor $A$ reflects the quality of the solvent. $A \leqslant 0$ represents a system of polymer 


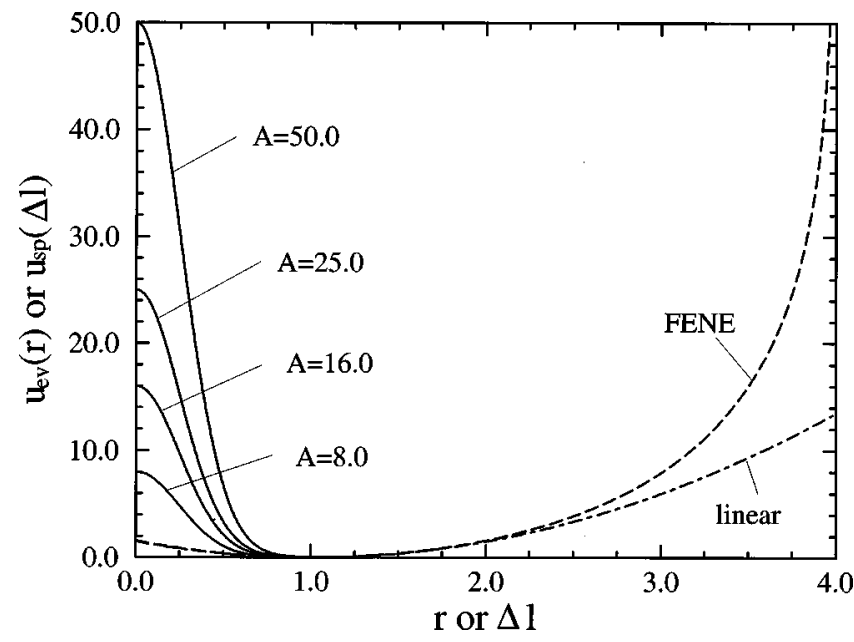

FIG. 1. Range and strength of the spring potential $u_{\mathrm{sp}}(\Delta l)$ and the EV potential $u_{\mathrm{ev}}(r)$, where $\Delta l=l-l_{0}, l$ is the distance between two linked beads, $l_{0}$ the natural length of the spring, and $r$ the distance between two unlinked beads. The dashed line is the FENE spring potential defined by Eq. (14) with $H=3.0, \Delta l_{\max }=3.0$, and $l_{0}=1.0$. It reduces to $u_{\mathrm{sp}}(\Delta l)=1.5 k_{B} T(\Delta l)^{2}$ at $\Delta l=0$ represented by the dot-dashed line. The solid lines represent the EV potential defined by Eq. (15) with $\sigma=0.25$ at different values of $A$. Energy is in $k_{B} T$ and the distances are in $l_{0}$ or $b$.

chains in a bad solvent, $A=0$ the chains in a $\theta$ solvent, and $A>0$ the chains in a good solvent.

The strength and the interaction range of the spring potential and the EV potential (on the left) are shown in Fig. 1. We can see that the FENE potential prevents the bead-bead bond length from becoming too elongated.

From the positions and forces we can calculate the specific viscosity of the system using the appropriate GreenKubo formula [36],

$$
\begin{aligned}
\eta_{\mathrm{sp}} \equiv & \frac{\eta-\eta_{s}}{\eta_{s}}=\frac{\beta}{\eta_{s} V} \int_{0}^{\infty}\left[\left\langle J^{\alpha \beta}(0) J^{\alpha \beta}(t)\right\rangle\right. \\
& \left.-\left\langle J_{s}^{\alpha \beta}(0) J_{s}^{\alpha \beta}(t)\right\rangle\right] d t,
\end{aligned}
$$

where $V$ is the volume of the system and $J^{\alpha \beta}$ is an offdiagonal component of the momentum flux tensor $\mathbf{J}$ which is related to the pressure tensor $\mathbf{P}$ by $\mathbf{J}=V \mathbf{P}$. The $(s-s)$ integral involving the second term on the right-hand side represents the contribution from the solvent alone. There are polymer and solvent contributions to $\mathbf{J}$ so that, decomposed into its components,

$$
\begin{aligned}
\left\langle J^{\alpha \beta}(0) J^{\alpha \beta}(t)\right\rangle= & \left\langle J_{p}^{\alpha \beta}(0) J_{p}^{\alpha \beta}(t)\right\rangle+\left\langle J_{p}^{\alpha \beta}(0) J_{0}^{\alpha \beta}(t)\right\rangle \\
& +\left\langle J_{0}^{\alpha \beta}(0) J_{p}^{\alpha \beta}(t)\right\rangle+\left\langle J_{0}^{\alpha \beta}(0) J_{0}^{\alpha \beta}(t)\right\rangle .
\end{aligned}
$$

The subscript $s$ indicates the momentum flux from the pure solvent, $p$ the contribution from the polymer potential parts, and 0 the contribution from the solvent part. Only the first $(p-p)$ polymer contribution in Eq. (17) is important in this context, since we assume in our model that there is no correlation between the polymer and the solvent. Therefore, the $(p-0)$ and $(0-p)$ terms are statistically zero. Also, we can assume the (0-0) and (s-s) correlation functions are identical. We therefore have only the ( $p-p)$ term left, which dominates anyway with increasing concentration, since it is the most slowly decaying correlation function [37,38],

$$
\eta_{\mathrm{sp}}=\frac{\beta}{\eta_{s}} \int_{0}^{\infty} G(t) d t
$$

where the correlation function is

$$
G(t) \equiv \frac{1}{V}\left\langle J_{p}^{\alpha \beta}(0) J_{p}^{\alpha \beta}(t)\right\rangle,
$$

with

$$
J_{p}^{\alpha \beta}=\sum_{i} \sum_{j \neq i} r_{i j \alpha} \mathbf{f}_{i j \beta},
$$

$\mathbf{f}_{i j}$ being the force exerted on bead $i$ by bead $j$ and $r_{i j \alpha}$ being the $\alpha$ component of the pair separation $r_{i j}$.

\section{COMPUTATIONAL DETAILS}

BD simulations were carried out for both $F 0$ and $F 1$ systems with varying EV strengths $A$, different chain lengths $N$, and at a series of concentrations. During the simulation the following quantities were set to unity for computational convenience: the thermal energy $k_{B} T$, the friction coefficient $\zeta$, mass of the bead $m$, viscosity of the solvent $\eta_{s}$, the rootmean-square bond length $b$ of the Rouse chain, and the natural spring bond length $l_{0}$ for the $F 1$ potential model. All other quantities are in reduced units. In such a case, the time step for the simulation is proportional to the mean-square random displacement of a bead,

$$
\Delta t=\frac{1}{2}\left\langle\left(\Delta R_{i \alpha}^{G}\right)^{2}\right\rangle .
$$

After several exploratory simulations, we chose $\left\langle\left(\Delta R_{i \alpha}^{G}\right)^{2}\right\rangle=0.05$ for most of the concentrations. However, for highly concentrated systems, i.e., $\rho>35.0,\left\langle\left(\Delta R_{i \alpha}^{G}\right)^{2}\right\rangle$ $=0.04$ was used to employ smaller time steps. The number of chains in the system was typically $N_{\mathrm{ch}}=20$ for dilute systems and set to different values from $N_{\mathrm{ch}}=40$ to $N_{\mathrm{ch}}=200$ for concentrated systems. A typical simulation that gave adequate statistics lasted for about four million time steps.

\section{RESULTS AND DISCUSSION}

Our model EV potential given in Eq. (15) had two adjustable parameters, which we call the affinity factor $A$ and the potential range $\sigma$. The former determines the quality of the solvent and the latter determines, among other things, the stiffness of the chain. To examine the behavior of flexible chains in solutions of finite concentration, we need to make sure that the parameters are suitably chosen so that our system represents flexible chains in a good solvent. We carried out exploratory simulations in the limit of infinite dilution to determine these parameters. Also, an appropriate value of $N$, the number of beads per chain, had to be chosen such that the chains were polymerlike and yet not too computationally expensive to calculate. 
TABLE I. The scaling exponent $\nu_{\eta}$ defined in Eq. (5) for the systems of $F 0$ spring potential Eq. (13) with different values of $A$ and $\sigma$.

\begin{tabular}{rcc}
\hline \hline \multicolumn{1}{c}{$A$} & $\sigma$ & $\nu$ \\
\hline 0.0 & 0.00 & $0.500 \pm 0.005$ \\
1.0 & 0.25 & $0.559 \pm 0.005$ \\
8.0 & 0.25 & $0.597 \pm 0.005$ \\
75.0 & 0.25 & $0.634 \pm 0.005$ \\
1.0 & 1.00 & $0.794 \pm 0.005$ \\
\hline \hline
\end{tabular}

The value of the scaling exponent $\nu$ in Eq. (2) reflects the stiffness of the chain. In a good solvent, $\nu>0.8$, indicates a stiff chain. We performed a number of simulations using the model $F 0$ potential type with different $A$ and $\sigma$ values. The values of the scaling exponent $\nu$ obtained from these simulations with the different potential parameters are listed in Table I. In a $\theta$ solvent, the calculated value of $\nu$ was, within statistical uncertainty, the Rouse value of 0.5 , as expected. For $\sigma=0.25$, as the affinity factor $A$ increases from 1.0 to 75.0 , the value of $\nu$ increased from 0.56 to 0.63 , reflecting the behavior of flexible chains as the quality of the solvent improves. For the case $A=1.0$ and $\sigma=1.0$ we have $\nu$ $=0.794$, which indicates that the chains were relatively stiff. The effect of $\sigma$ on the scaling behavior is shown clearly in Figs. 2(a) and 2(b). We used $\sigma=0.25$ in all subsequent simulations to ensure sufficiently flexible chains throughout this study.

In the following sections we first look at the influences of solvent quality and chain length on the properties of the polymer coils in the limit $\rho \rightarrow 0$. Then we examine the changes in these model polymer solutions brought about by increasing concentration.

\section{A. Behavior at infinite dilution}

In the limit of infinite dilution, i.e., $\rho \rightarrow 0$, there are no interactions between unlinked beads under $\theta$ conditions and a flexible polymer chain therefore assumes a Gaussian conformational distribution between any two beads $m$ and $n$ on the chain. In a good solvent, since $\rho \rightarrow 0$, there are no interchain excluded-volume interactions so that in this limit the deviation from the Gaussian chain behavior is caused solely by intrachain EV interactions. These intrachain EV repulsions push the beads further apart so that the chains form larger coils than in a $\theta$ solvent. The longer the chain, the more pronounced this intrachain EV effect. Figures 3(a) and 3(b) show the distribution of the radius of gyration, $W\left(R_{\mathrm{gyr}}\right)$, as a function of chain length for both systems under $\theta$ and good solvent conditions. For a given polymer the coil size in a good solvent is larger than in the $\theta$ solvent. This difference in size is enhanced as the number of beads per chain $N$ increases, since the longer chains presumably have greater scope for expansion. These effects are also clearly shown in the data listed in Table II.

First we look at the simulation results of the root-meansquare (rms) bond length $b$, listed in Table II. In a $\theta$ solvent (i.e., $A=0.0$ ) the quantity $b$ is independent of $N$ for both classes of systems. We found $b=1.003 \pm 0.001$ for the $F 0$ series and $b=1.535 \pm 0.001$ for the $F 1$ series. As the quality
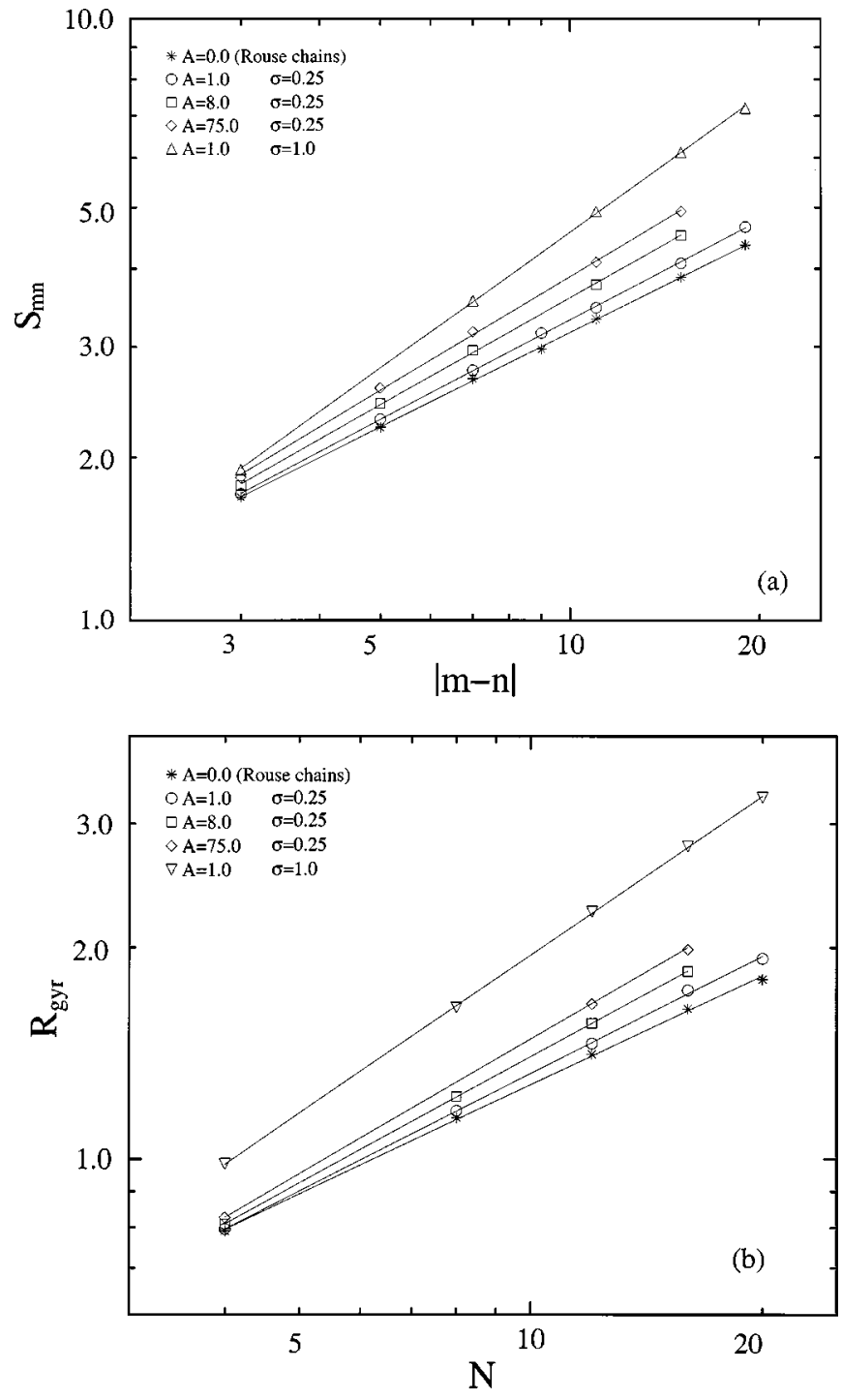

FIG. 2. Effects of $u_{\mathrm{ev}}(r)$ with different $A$ and $\sigma$ on the scaling behavior of the $F 0$ system in the limit $\rho \rightarrow 0$. (a) Root-mean-square distance between two beads $m$ and $n$ on the same chain, $S_{m n}$, as a function of $|m-n|$; (b) Radius of gyration $R_{\text {gyr }}$ as a function of number of beads per chain, $N$.

of the solvent became better, i.e., as $A$ increased, the intrachain EV repulsions caused the springs to expand slightly. The larger the value of $A$ the greater the elongation and also, for a given value of $A$, the elongation increased with chain length, $N$. Nevertheless, on the whole, the increase in bond length $b$ was small, i.e., $<8 \%$ for the $F 0$ series and $<3 \%$ for the $F 1$ series. This suggests that the $F 1$ spring potential was a more realistic representation of the polymer than the Rouse interactions, presumably because segments of a real polymer chain are not so easily deformed.

Within the statistical uncertainty, the results of our simulations are in agreement with the scaling laws [Eqs. (1) and (2)] that describe the dimensions of the polymer coils in the limit of infinite dilution. The reduced quantities that represent the chain dimensions are $S_{\mathrm{ee}}^{*} \equiv S_{\mathrm{ee}} / b, S_{\mathrm{me}}^{*} \equiv S_{\mathrm{me}} / b$, and $R_{\mathrm{gyr}}^{*} \equiv R_{\mathrm{gyr}} / b$. These quantities for various system parameters are listed in Table II, as well as the scaling exponent $\nu$ calculated from them. The average deviation for the values of these quantities obtained from simulation is \pm 0.006 . Fig- 

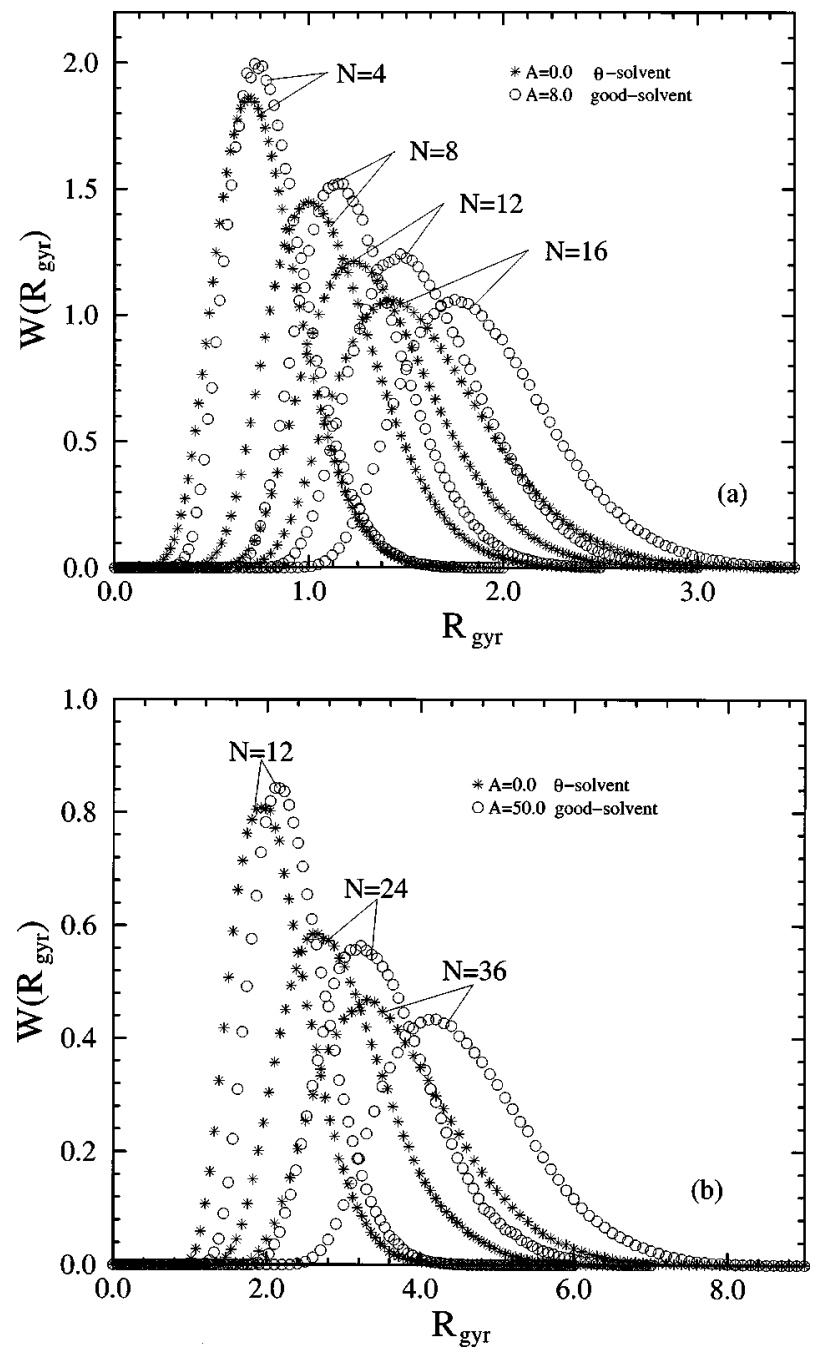

FIG. 3. Swelling of the polymer chains in a good solvent as represented by the probability distribution of the radius of gyration, $W\left(R_{\mathrm{gyr}}\right)$, as a function of number of beads per chain, $N$, in different solvents. (a) $F 0$ system and (b) $F 1$ system.

ures 2(a) and 4(a) give the root-mean-square distance between the two beads $m$ and $n, S_{m n}$, as a function of $|n-m|$, i.e., for end-to-end distance $S_{\text {ee }}|n-m|=N-1$ and for middle-to-end distance $S_{\text {me }}|n-m|=N / 2-1$. Figures 2(b) and 4(b) show the root-mean-square radius of gyration as a function of $N$.

For the system of type $F 0$ with $N=4$, the reduced middleto-end distance $S_{\text {me }}^{*}$ decreased slightly as the solvent became better, which indicates that the chain was perhaps too short for the observance of true scaling behavior, and therefore we calculated $\nu$ only using systems with $N \geqslant 8$. For the $F 0$ series the values of $\nu$ calculated from $S_{\mathrm{ee}}^{*}$ and $S_{\mathrm{me}}^{*}$ are statistically indistinguishable, while the values of $\nu$ calculated from $R_{\mathrm{gyr}}^{*}$ are somewhat larger, e.g., for the $F 0$ system with $A=8.0$ we found $\nu=0.597$ from $R_{\mathrm{gyr}}^{*}$ but $\nu=0.572$ from $S_{\mathrm{ee}}^{*}$. We assume that this difference is caused by the limited value of the chain length $N$. As the chains became longer the difference between the $\nu$ values calculated from $S_{\text {ee }}^{*}$ and from $R_{\mathrm{gyr}}^{*}$ was reduced, as was the case of $F 1$ series. Also for the $F 1$ series in good solvents, as the chains became longer the $\nu$ values calculated from $S_{\text {ee }}^{*}$ were seen to be larger than those calcu-
TABLE II. The scaling exponent $\nu$ defined in Eqs. (1) and (2) calculated from the reduced chain dimensions. $b$ is the root-meansquare bond length and the reduced quantities are defined by $S_{\text {ee }}^{*}$ $\equiv S_{\mathrm{ee}} / b, S_{\mathrm{me}}^{*} \equiv S_{\mathrm{me}} / b$, and $R_{\mathrm{gyr}}^{*} \equiv R_{\mathrm{gyr}} . r$ represents the correlation coefficient of the power-law regression.

\begin{tabular}{|c|c|c|c|c|c|c|c|c|}
\hline$F 0$ & $N$ & 4 & 8 & 12 & 16 & 20 & $\nu$ & $r$ \\
\hline \multirow[t]{4}{*}{$A=0.0$} & $b$ & 1.002 & 1.002 & 1.002 & 1.003 & 1.004 & & \\
\hline & $S_{\mathrm{ee}}^{*}$ & 1.732 & 2.664 & 3.321 & 3.872 & 4.356 & 0.500 & 0.9999 \\
\hline & $S_{\mathrm{me}}^{*}$ & 1.000 & 1.731 & 2.232 & 2.669 & 2.972 & 0.497 & 0.9995 \\
\hline & $R_{\mathrm{gyr}}^{*}$ & 0.790 & 1.144 & 1.408 & 1.635 & 1.803 & 0.500 & 0.9996 \\
\hline \multirow[t]{4}{*}{$A=1.0$} & $b$ & 1.010 & 1.016 & 1.019 & 1.021 & 1.022 & & \\
\hline & $S_{\mathrm{ee}}^{*}$ & 1.748 & 2.722 & 3.464 & 4.097 & 4.658 & 0.531 & 0.9999 \\
\hline & $S_{\mathrm{me}}^{*}$ & 0.995 & 1.756 & 2.299 & 2.752 & 3.154 & 0.533 & 0.9999 \\
\hline & $R_{\mathrm{gyr}}^{*}$ & 0.795 & 1.170 & 1.459 & 1.738 & 1.930 & 0.555 & 0.9999 \\
\hline \multirow[t]{4}{*}{$A=8.0$} & $b$ & 1.031 & 1.045 & 1.049 & 1.051 & & & \\
\hline & $S_{\mathrm{ee}}^{*}$ & 1.797 & 2.905 & 3.769 & 4.514 & & 0.572 & 0.9999 \\
\hline & $S_{\mathrm{me}}^{*}$ & 0.992 & 1.816 & 2.435 & 2.961 & & 0.571 & 0.9999 \\
\hline & $R_{\mathrm{gyr}}^{*}$ & 0.808 & 1.227 & 1.560 & 1.851 & & 0.597 & 0.9999 \\
\hline \multirow[t]{4}{*}{$A=75.0$} & $b$ & 1.058 & & 1.080 & 1.082 & & & \\
\hline & $S_{\mathrm{ee}}^{*}$ & 1.860 & & 4.093 & 4.929 & & 0.606 & 0.9999 \\
\hline & $S_{\mathrm{me}}^{*}$ & 0.982 & & 2.578 & 3.171 & & 0.601 & 0.9999 \\
\hline & $R_{\mathrm{gyr}}^{*}$ & 0.826 & & 1.662 & 1.987 & & 0.634 & 0.9999 \\
\hline$F 1$ & $N$ & & & 12 & 24 & 36 & $\nu$ & $r$ \\
\hline \multirow[t]{4}{*}{$A=0.0$} & $b$ & & & 1.534 & 1.534 & 1.536 & & \\
\hline & $S_{\mathrm{ee}}^{*}$ & & & 3.327 & 4.763 & 5.945 & 0.500 & 0.9998 \\
\hline & $S_{\mathrm{me}}^{*}$ & & & 2.237 & 3.290 & 4.123 & 0.498 & 0.9999 \\
\hline & $R_{\mathrm{gyr}}^{*}$ & & & 1.412 & 1.988 & 2.454 & 0.502 & 0.9999 \\
\hline \multirow[t]{4}{*}{$A=8.0$} & $b$ & & & 1.545 & 1.552 & 1.555 & & \\
\hline & $S_{\mathrm{ee}}^{*}$ & & & 3.529 & 5.316 & 6.682 & 0.552 & 0.9999 \\
\hline & $S_{\mathrm{me}}^{*}$ & & & 2.322 & 3.584 & 4.564 & 0.552 & 0.9999 \\
\hline & $R_{\mathrm{gyr}}^{*}$ & & & 1.480 & 2.180 & 2.728 & 0.557 & 0.9999 \\
\hline \multirow[t]{4}{*}{$A=16.0$} & $b$ & & & 1.555 & 1.556 & 1.560 & & \\
\hline & $S_{\mathrm{ee}}^{*}$ & & & 3.605 & 5.448 & 7.010 & 0.573 & 0.9998 \\
\hline & $S_{\mathrm{me}}^{*}$ & & & 2.357 & 3.657 & 4.712 & 0.565 & 0.9999 \\
\hline & $R_{\mathrm{gyr}}^{*}$ & & & 1.504 & 1.227 & 2.835 & 0.576 & 0.9999 \\
\hline \multirow[t]{4}{*}{$A=25.0$} & $b$ & & & 1.556 & 1.559 & 1.563 & & \\
\hline & $S_{\mathrm{ee}}^{*}$ & & & 3.639 & 5.518 & 7.149 & 0.581 & 0.9997 \\
\hline & $S_{\mathrm{me}}^{*}$ & & & 2.372 & 3.696 & 4.795 & 0.574 & 0.9999 \\
\hline & $R_{\mathrm{gyr}}^{*}$ & & & 1.516 & 2.255 & 2.883 & 0.584 & 0.9999 \\
\hline \multirow[t]{4}{*}{$A=30.0$} & $b$ & & & 1.557 & 1.560 & 1.564 & & \\
\hline & $S_{\mathrm{ee}}^{*}$ & & & 3.661 & 5.578 & 7.199 & 0.583 & 0.9999 \\
\hline & $S_{\mathrm{me}}^{*}$ & & & 2.383 & 3.727 & 4.827 & 0.576 & 0.9999 \\
\hline & $R_{\mathrm{gyr}}^{*}$ & & & 1.523 & 2.272 & 2.903 & 0.586 & 0.9999 \\
\hline \multirow[t]{4}{*}{$A=50.0$} & $b$ & & & 1.560 & 1.563 & 1.568 & & \\
\hline & $S_{\mathrm{ee}}^{*}$ & & & 3.699 & 5.656 & 7.339 & 0.590 & 0.9998 \\
\hline & $S_{\mathrm{me}}^{*}$ & & & 2.400 & 3.772 & 4.916 & 0.584 & 0.9999 \\
\hline & $R_{\mathrm{gyr}}^{*}$ & & & 1.535 & 2.300 & 2.952 & 0.594 & 0.9999 \\
\hline
\end{tabular}

lated from $S_{\text {me }}^{*}$, which seems to suggest that this end-chain effect is more pronounced in a good solvent.

We will use the average of the $\nu$ values determined by $S_{\mathrm{me}}^{*}, S_{\mathrm{ee}}^{*}$, and $R_{\mathrm{gyr}}^{*}$ as the index for solvent quality. In a $\theta$ solvent, for both $F 0$ and $F 1$ systems, the average value of $\nu$ is $0.500 \pm 0.003$, indicating Rouse behavior. As the affinity factor $A$ increases we find that $\nu$ increases above 0.5 , indicating a better solvent. The $F 0$ system with $A=8.0$ gives a 

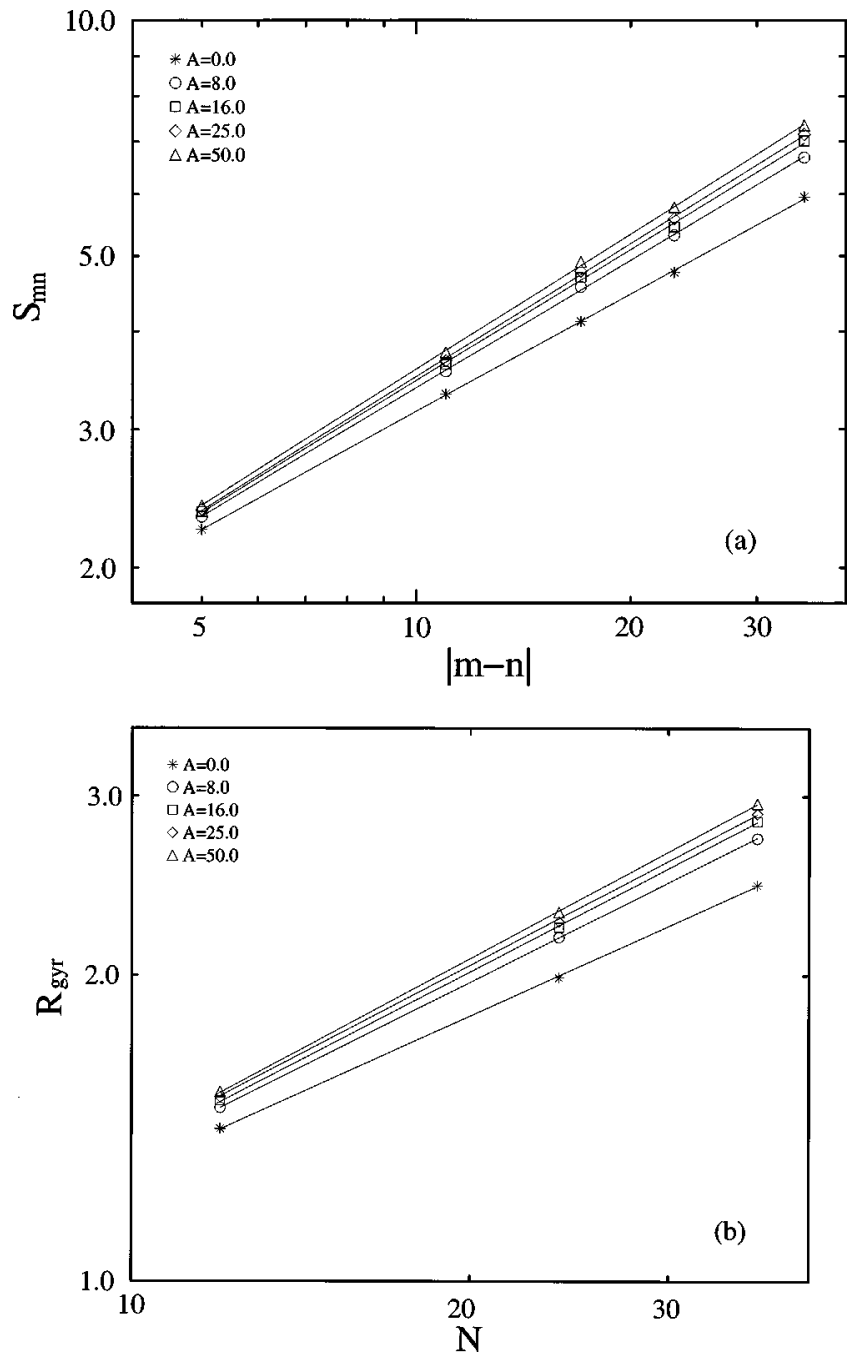

FIG. 4. Effects of $u_{\mathrm{ev}}(r)$ with $\sigma=0.25$ and different values of $A$ on the scaling behavior of the $F 1$ system in the limit $\rho \rightarrow 0$. (a) Root-mean-square distance between two beads $m$ and $n$ on the same chain, $S_{m n}$, as a function of $|m-n|$; (b) Radius of gyration $R_{\text {gyr }}$ as a function of number of beads per chain, $N$.

reasonably good solvent with an average $\nu=0.58 \pm 0.01$. The $F 1$ system, using $A=25.0$ also gives a reasonably good solvent with an average $\nu=0.58 \pm 0.01$. Close to $\theta$ conditions a small increase in $A$ improves the solvent quality signifi-

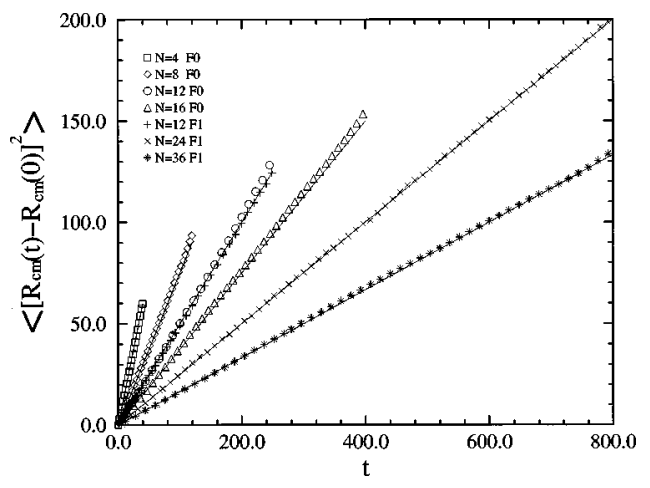

FIG. 5. Mean-square displacement of the center of mass $\left\langle\left[R_{\mathrm{cm}}(t)-R_{\mathrm{cm}}(0)\right]^{2}\right\rangle$ as a function of time $t$ for an $F 0$ system with $A=8.0$ and an $F 1$ system with $A=25.0$ for different values of $N$.
TABLE III. The diffusion coefficient of the center of mass $D_{\mathrm{cm}}$ defined by Eq. (4) as a function of $N$ and $A . D_{\mathrm{cm}}^{\mathrm{RS}}=1 / N$ is the Rouse value.

\begin{tabular}{lcccccc}
\hline \hline \multicolumn{1}{c}{$F 0$} & $N$ & 4 & 8 & 12 & 16 & 20 \\
\hline$D_{\mathrm{cm}}^{\mathrm{RS}}$ & & 0.250 & 0.125 & 0.0833 & 0.0625 & 0.0500 \\
$A=0.0$ & & 0.256 & 0.127 & 0.0832 & 0.0628 & 0.0496 \\
$A=1.0$ & & 0.250 & 0.126 & 0.0837 & 0.0619 & 0.0497 \\
$A=8.0$ & & 0.251 & 0.131 & 0.0841 & 0.0631 & \\
$A=75.0$ & & 0.245 & & 0.0840 & 0.0624 & \\
\multicolumn{1}{c}{$F 1$} & $N$ & & & 12 & 24 & 36 \\
\hline$D_{\mathrm{cm}}^{\mathrm{RS}}$ & & & & 0.0833 & 0.0417 & 0.0278 \\
$A=8.0$ & & & & 0.0838 & 0.0419 & 0.0281 \\
$A=16.0$ & & & & 0.0829 & 0.0423 & 0.0288 \\
$A=25.0$ & & & & 0.0836 & 0.0416 & 0.0275 \\
$A=30.0$ & & & & 0.0832 & 0.0417 & 0.0279 \\
$A=50.0$ & & & & 0.0833 & 0.0419 & 0.0285 \\
\hline \hline
\end{tabular}

cantly, while in the good solvent range a similar increase in $A$ causes only a small increase in the value of $\nu$.

Figure 5 shows the mean-square displacement of the center of mass $\left\langle\left[\mathbf{R}_{\mathrm{cm}}(t)-\mathbf{R}_{\mathrm{cm}}(0)\right]^{2}\right\rangle$ as function of time for two different systems, $F 0$ with $A=8.0$ and $F 1$ with $A=25.0$. The self-diffusion coefficient $D_{\mathrm{cm}}$ was calculated from Eq. (4) and they show a $D_{\mathrm{cm}} \propto N^{-1}$ dependence for all the systems. The values of $D_{\mathrm{cm}}$ obtained from the simulations are listed in Table III. In a $\theta$ solvent, because we are in the free-draining limit, $\widetilde{\nu}_{D}$ takes the form $1 / N$, the value of Rouse chains, as expected. Significantly, this data also shows that solvent quality does not influence this $N^{-1}$ dependence. Moreover, despite the fact that the bond length $b$ of $F 1$ system is longer than that of $F 0$ system with the same $N$, the value of $D_{\mathrm{cm}}$ for the two systems are, within statistical uncertainty, the same. These results show that in the infinite dilution limit the diffusion behavior of the center of mass is independent of the interaction law between the beads.

The scaling relations between intrinsic viscosity $[\eta]$ and number of beads per chain $N$ given by Eq. (5) for different interaction parameters are shown in Fig. 6. The values of the

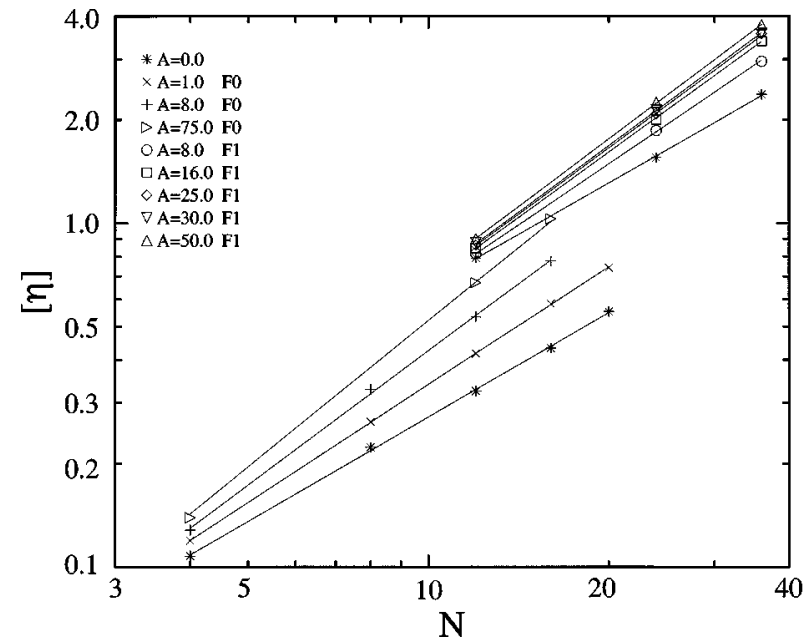

FIG. 6. The intrinsic viscosity $[\eta]$ as a function of $N$ in different solvents for both $F 0$ and $F 1$ systems. 
TABLE IV. The scaling exponent $\nu_{\eta}$ defined in Eq. (5) for the intrinsic viscosity. $\Delta \nu_{\eta}=\nu_{\eta}(A)-\nu_{\eta}(0)$.

\begin{tabular}{rrrrrl}
\hline \hline \multicolumn{1}{c}{$A$} & $F 0$ & & & \multicolumn{1}{c}{$F 1$} \\
& $\nu_{\eta}$ & $\Delta \nu_{\eta}$ & \multicolumn{1}{c}{$A$} & \multicolumn{1}{c}{$\nu_{\eta}$} & $\Delta \nu_{\eta}$ \\
\hline 0.0 & 1.01 & 0.00 & 0.0 & 0.99 & 0.0 \\
1.0 & 1.14 & 0.13 & 8.0 & 1.18 & 0.19 \\
8.0 & 1.29 & 0.28 & 16.0 & 1.26 & 0.27 \\
75.0 & 1.41 & 0.40 & 25.0 & 1.28 & 0.29 \\
& & & 30.0 & 1.29 & 0.30 \\
& & 50.0 & 1.31 & 0.31 \\
\hline \hline
\end{tabular}

scaling exponent $\nu_{\eta}$ were calculated and they are listed in Table IV. The scaling exponent $\nu_{\eta} \geqslant 1$, which is expected, since we are in the free-draining limit. Although the absolute values of $\nu_{\eta}$ are therefore physically unrealistic, it is interesting to look at the quantity $\Delta \nu_{\eta} \equiv \nu_{\eta}-\nu_{\eta}(\theta)$. For flexible real polymers $\nu_{\eta}$ varies in the range $0.5-0.8$ in good solvents with $\nu_{\eta}(\theta)=0.5$ in $\theta$ solvent, therefore giving $\Delta \nu_{\eta}$ in the range $0.0 \sim 0.3$. Our results for both series fall in this range, which suggests that in the infinite dilution limit the quantity $\Delta \nu_{\eta}$ is only weakly dependent on the presence of manybody hydrodynamic interactions, and the MHI contribution to $\nu_{\eta}$ from a polymer chain in a good solvent is the similar to that for a polymer in a $\theta$ solvent.

\section{B. Behavior at finite concentrations}

At finite polymer concentration, the interchain EV forces become increasingly important. There are three distinguishable regimes. In the dilute region, because of the interchain repulsions, the polymer chains avoid overlap on approach by shrinking into slightly smaller coils. As a result, the polymers behave like isolated coils that only interact occasionally. In the concentrated region, the system becomes more crowded and the coils are forced to overlap for extended periods. They begin to interpenetrate, which reduces the net effect of the intrachain repulsion energy. In the highly concentrated region, the density fluctuations are greatly suppressed and each bead is surrounded by an increasing number of beads from other chains, reducing its ability to distinguish between beads from the same chain and those from other chains. The segment-segment interactions tend to become "balanced out" so that when the system reaches the melt state the chains become conformationally ideal again. Our simulation results show these three concentration re-

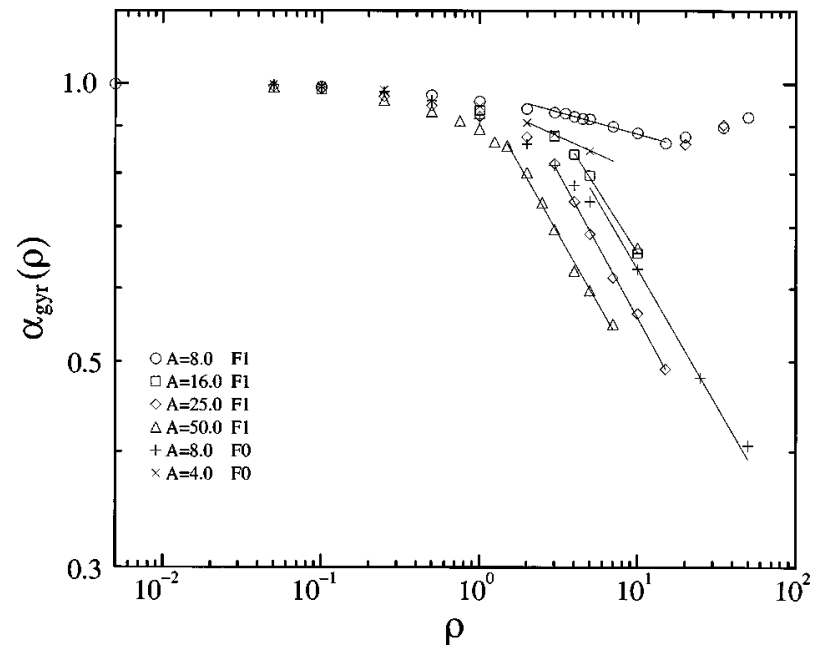

FIG. 7. Normalized radius of gyration $\alpha_{\text {gyr }}(\rho)$ $\equiv R_{\mathrm{gyr}}(\rho) / R_{\mathrm{gyr}}(0)$ as a function of number density of beads $\rho$ in different solvents for both the $F 0$ and $F 1$ systems with $N=12$.

gions clearly, and below we demonstrate how the key properties change as functions of concentration. However, we must bear in mind that in our model the entanglement effect (which is caused by physical connectivity of the beads on a chain) is not included completely and in the highly concentrated region we expect the polymers to behave like Rouse chains, which is acceptable for the chain conformational distribution, but not for the dynamical properties.

\section{Radius of gyration}

The change in the size of a typical polymer chain as concentration increases is conveniently characterized by the ratio $\alpha_{\text {gyr }}(\rho) \equiv R_{\text {gyr }}(\rho) / R_{\text {gyr }}(0)$, which is shown as a function of number density of beads $\rho$ in Fig. 7. First we consider the $F 1$ class system in a moderate solvent (i.e., $A=8.0$ ). In the dilute region $(\rho<2.0)$, the radius of gyration of the coil $R_{\mathrm{gyr}}$ drops steadily as $\rho$ increases. Entering the concentrated region (ca. $2.0<\rho<15.0$ ), $R_{\text {gyr }}$ decreases more dramatically and follows the scaling law $\alpha_{\mathrm{gyr}} \propto \rho^{\kappa}$. In the highly concentrated region, $\rho>15.0, R_{\text {gyr }}$ increases with $\rho$, indicating a return to Rouse-like conformational statistics. This is the general pattern for the $F 1$ system in different solvents and, in fact, in better solvents (i.e., increasing magnitude of $A$ ) the changes are even more pronounced (e.g., for $A=16$ and 25) and takes place at lower $\rho$ for larger $A$ values. For the $F 0$ system, since $l_{0}=0$, the concentrated region is more ex-

TABLE V. The scaling exponents of $\rho$ in the concentrated region defined by Eqs. (9)-(11) in different solvents. $r$ is the correlation coefficient of the power-law regression. $\alpha_{\mathrm{gyr}} \propto \rho^{\kappa}, \alpha_{\mathrm{cm}} \propto \rho^{\kappa} D, \eta_{r} \propto \rho^{\kappa} \eta$, and $\eta_{\mathrm{sp}}$ $\propto \rho^{\kappa}$ sp.

\begin{tabular}{cccccccccc}
\hline \hline & & $\kappa$ & $r$ & $\kappa_{D}$ & $r$ & $\kappa_{\eta}$ & $r$ & $\kappa_{\mathrm{sp}}$ & $r$ \\
\hline$F 0$ & $A=4.0$ & -0.08 & -0.998 & & & 0.44 & 0.999 & 0.89 & 0.998 \\
& $A=8.0$ & -0.30 & 0.998 & -0.17 & 0.996 & 0.54 & 0.998 & 0.85 & 0.999 \\
$F 1$ & $A=8.0$ & -0.05 & -0.994 & -0.16 & -0.999 & 0.90 & 0.999 & 1.06 & 0.999 \\
& $A=16.0$ & -0.27 & -0.999 & -0.41 & -0.999 & 1.00 & 0.999 & 1.16 & 0.999 \\
& $A=25.0$ & -0.32 & -0.999 & -0.77 & -0.996 & 1.17 & 0.999 & 1.26 & 0.998 \\
& $A=50.0$ & -0.31 & -0.995 & -1.70 & -0.998 & 1.32 & 0.994 & 1.45 & 0.996 \\
\hline \hline
\end{tabular}




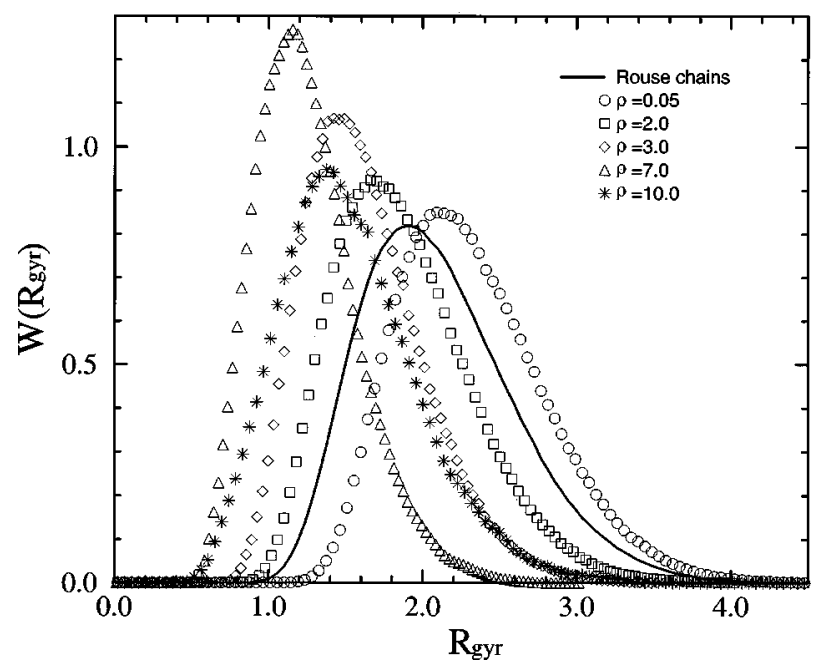

FIG. 8. Distribution of the radius of gyration $W\left(R_{\mathrm{gyr}}\right)$ as a function of $\rho$ for the $F 1$ system with $A=50.0$ and $N=12$.

tended than that of the $F 1$ system and we do not see an increase in the value of $\alpha_{\text {gyr }}(\rho)$ in the density range covered in our simulations.

Both of the potential forms, $F 0$ and $F 1$, show scaling behavior in the concentrated region with the analytic form, $\alpha_{\text {gyr }} \propto \rho^{\kappa}$. The scaling exponent $\kappa$ depends on solvent quality, values of which are listed in Table V. In good solvents, the scaling theory predicts that $\kappa=(1-2 \nu) / 2(3 \nu-1)=-1.25$ [30]. Unfortunately, there are only a few experimental results available. Some confirm the theory $[40,41]$, whereas others give a smaller value $\kappa=-0.08$ [42]. Our results show a much faster decrease in $R_{\mathrm{gyr}}$ as concentration increases and the data suggest that $\kappa \approx \nu / 2 \approx 0.3$. The simulations of Browstow and Drewmak, also with only EV repulsion, gave $\kappa$ $=0.2$ [43]. We suggest that this more dramatic decrease in chain dimension with increasing concentration of the solution is caused by the lack of topological entanglement in our model. Additional topological constraints would restrict the degree of shrinkage of the chains with increasing concentration.

The return to a Rouse-like chain conformational distribution in the concentrated region for the $F 1$ system can be seen in more detail in the change in the probability distribution function of $R_{\text {gyr }}$ with $\rho$ as shown in Fig. 8. As $\rho$ increases the polymer coils become smaller and the distribution curve shifts to the left until the system enters the highly concentrated region where the distribution curve starts to move back to the right toward the distribution of a flexible coil in a $\theta$ solvent.

The rms bond length $b$ also changes with increasing $\rho$. Table VI gives the value of $b$ as a function of $\rho$ for the $F 1$ system with $A=25.0$. As we can see, $b$ decreases as $\rho$ increases, then achieves a minimum and begins to increase again, indicating the return to Rouse chain behavior.

\section{Self-diffusion coefficient}

The ratio of the self-diffusion coefficient to its value in the zero density limit, $\alpha_{\mathrm{cm}}$, as a function of $\rho$ is shown in Fig. 9. Below the highly concentrated region, as $\rho$ increases, $D_{\mathrm{cm}}$ decreases, gradually at first in the dilute region and then
TABLE VI. The bond length $b$ as a function of $\rho$ for the $F 1$ system with $A=25.0$.

\begin{tabular}{llllll}
\hline \hline$\rho$ & $\rightarrow 0$ & 0.05 & 0.10 & 0.25 & 0.50 \\
$b$ & 1.5561 & 1.5531 & 1.5502 & 1.5410 & 1.5265 \\
$\rho$ & 1.00 & 2.00 & 3.00 & 4.00 & 5.00 \\
$b$ & 1.5030 & 1.4461 & 1.3510 & 1.2255 & 1.1098 \\
$\rho$ & 7.00 & 10.00 & 15.00 & 20.00 & 35.00 \\
$b$ & 0.9773 & 0.9261 & 0.6730 & 1.4690 & 1.4856 \\
\hline \hline
\end{tabular}

more steeply in the concentrated region, where $D_{\mathrm{cm}}$ follows the scaling law, $\alpha_{\mathrm{cm}} \propto \rho^{\kappa} D$. The values of the scaling exponent $\kappa_{D}$ obtained from the simulation data are presented in Table V. Again, the value of the exponent depends on solvent quality, with a better solvent giving a more negative value of $\kappa_{D}$. In a good solvent the value of $\kappa_{D}$ predicted by scaling theory is -1.75 , which has been confirmed by experiment [44]. One of our $F 1$ systems with $A=50.0$, has a similar value $\kappa_{D}=-1.70$. In the highly concentrated region, since there are no topological constraints in our systems, the value of $D_{\mathrm{cm}}$ starts to increase as a result of the decreasing impact of the EV effect, approaching the value characteristic of a Rouse chain. This unrealistic feature is again caused by the lack of physical entanglement.

\section{Viscosity}

The experimental results for various real polymer-solvent systems show that the relative viscosity $\eta_{r}$ can be described by a stretched exponential function of $\rho, \eta_{r}=\exp \left(a \rho^{x}\right)$, in the dilute region, where $a$ is a constant typical of each polymer, and by a power law, $\eta_{r} \propto \rho^{\kappa} \eta$ in the concentrated region [39]. The values of $\eta_{r}$ obtained from our simulations as function of the coil-overlap parameter $[\eta] \rho$ are shown in Fig. 10 . We reproduced the experimental trends in that the data in the dilute region follow a stretched exponential form and in the concentrated region a power law. Table $\mathrm{V}$ shows that the value of exponent $\kappa_{\eta}$ depends on solvent quality. In the highly concentrated region $\eta_{r}$ becomes less sensitive to $\rho$.

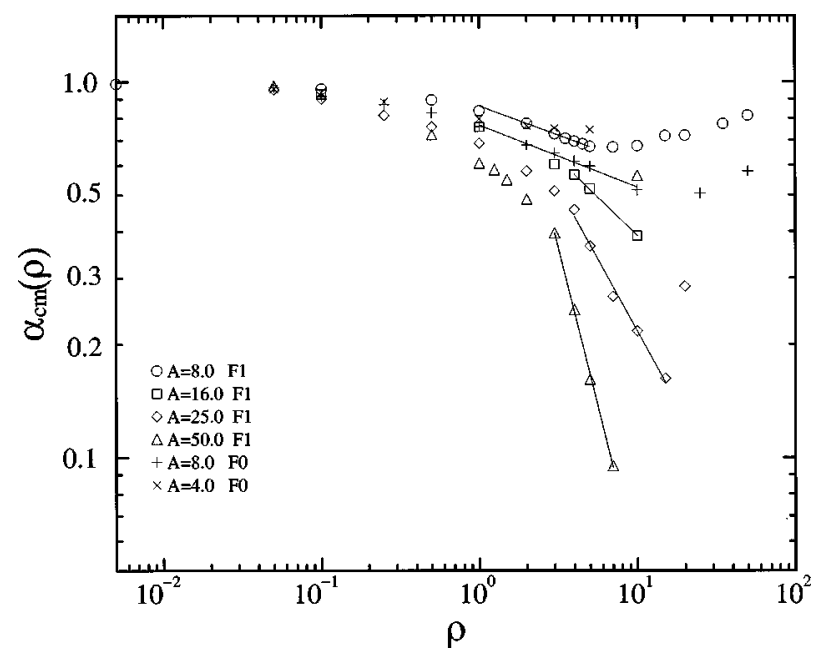

FIG. 9. Center-of-mass self-diffusion coefficient $D_{\mathrm{cm}}$ as a function of number density of beads $\rho$ in different solvents for both the $F 0$ and $F 1$ systems with $N=12$. 


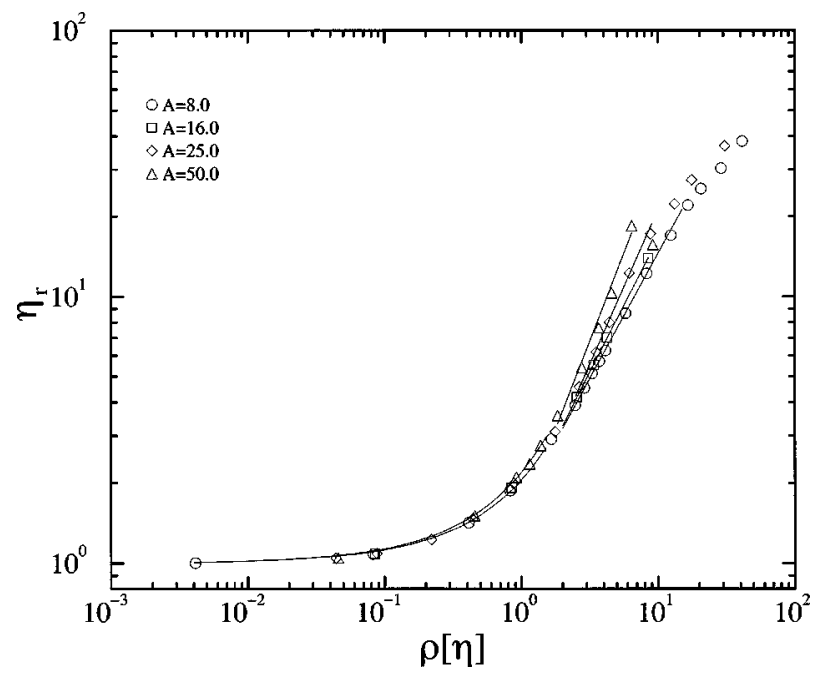

FIG. 10. Relative viscosity $\eta_{r}$ as a function of coil-overlap parameter $\rho[\eta]$ for the $F 1$ system with $N=12$ in different solvents.

Figure 11 shows the specific viscosity, $\eta_{\mathrm{sp}}$, for the $F 1$ system with $N-12$ as a function of coil-overlap parameter, $[\eta] \rho$, which is equivalent to $\rho / \rho^{*}$. The thick line in the figure is the relationship $\eta_{\mathrm{sp}}=[\eta] \rho$ that corresponds to Rouse behavior. In the dilute region the chains behave essentially as Rouse chains. As the solution becomes more concentrated, deviations begin to occur. (The crossover point from the dilute to the concentrated region is about $\rho[\eta] \approx 0.6)$. In the concentrated region the scaling law is found to be $\eta_{\mathrm{sp}}$

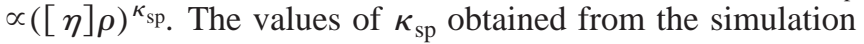
data are given in Table V. The better the solvent the larger the value of $\kappa_{\mathrm{sp}}$, although these values are much smaller than the value $3 / 3 \nu-1=3.75$ predicted by scaling theory [30]. In the highly concentrated region $\eta_{\mathrm{sp}}$ decreases, as the net effects of the excluded-volume repulsion decreases, and we return to Rouse-like behavior.

The effect of excluded-volume forces on the viscosity of the solution can be seen in the quantity, $\eta_{\mathrm{ev}}$, a measure of the excluded-volume contribution to the viscosity,

$$
\eta_{\mathrm{ev}} \equiv\left(\eta_{\mathrm{sp}}-\eta_{\mathrm{sp}}^{\mathrm{RS}}\right) / \rho[\eta]
$$

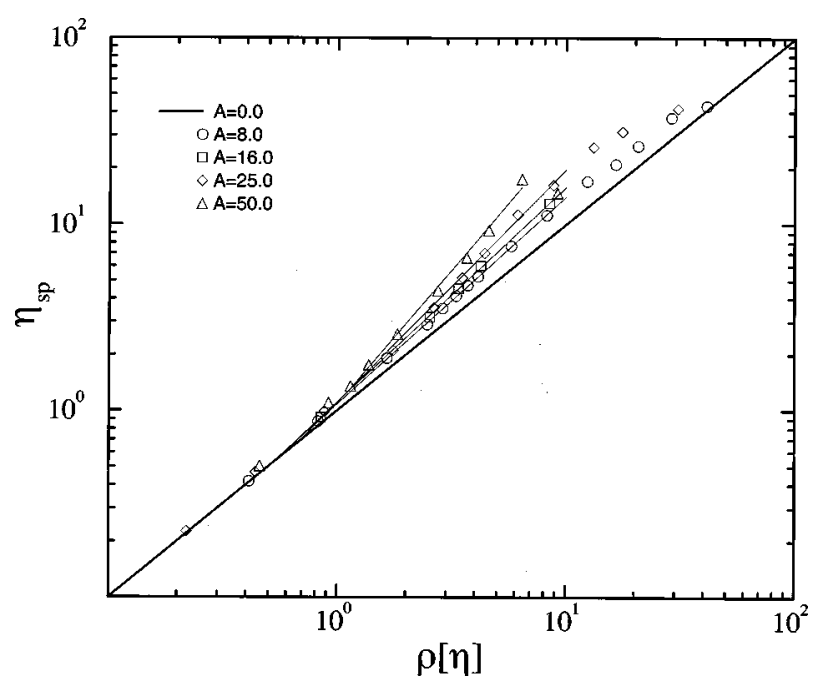

FIG. 11. Specific viscosity $\eta_{\mathrm{sp}}$ as a function of coil-overlap parameter $\rho[\eta]$ for an $F 1$ system with $N=12$ in different solvents.

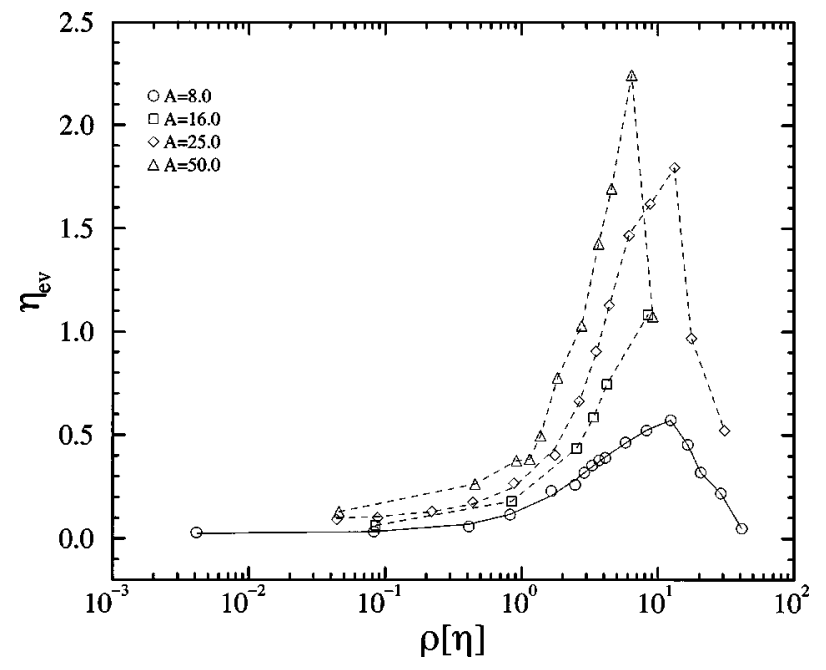

FIG. 12. EV contribution to viscosity, $\eta_{\mathrm{ev}}$, defined by Eq. (23), as a function of coil-overlap parameter $\rho[\eta]$ for the $F 1$ system with $N=12$ in different solvents.

where the Rouse value $\eta_{\mathrm{sp}}^{\mathrm{RS}}=N b^{2} / 36$. Since the bond length $b$ is a function of $\rho, \eta_{\mathrm{sp}}^{\mathrm{RS}}$ also depends on $\rho$. Figure 12 shows $\eta_{\mathrm{ev}}$ as function of the coil-overlap parameter. In the dilute region the interchain EV forces are small and the viscosity of the system is close to the Rouse value. In the concentrated region the interchain $\mathrm{EV}$ forces dominate and the viscosity increases markedly with density. As the systems become highly concentrated, the net effects of the EV forces begin to disappear and viscosity decreases toward that of the Rouse chain again. This latter unrealistic feature is again caused by partially neglecting the topological constraints of the chains using this coarse-grained model.

\section{CONCLUSIONS}

A model excluded-volume potential of the form $\beta u_{\mathrm{ev}}(r)$ $=A \exp \left(-r^{2} / 2 \sigma^{2}\right)$ with $\sigma=0.25$ and various values of $A$ is shown to give a reasonable description of the segmentsegment interactions for flexible polymers in solution over a wide concentration range. The simulation results give a clear picture of how the segment-segment interactions contribute to both static and dynamical properties of a model polymer solution and how these properties change as the system becomes increasingly concentrated. Comparisons with experiments reveal the strengths and limitations of our model.

In the limit of infinite dilution the static and dynamical properties agree quite well with theoretical predictions. In addition, the model exhibits the correct behavior for the chain dimensions in the whole concentration range as $\rho$ increases. In the dilute region the coil size decreases slowly, in the concentrated region the shrinkage of the coil follows a scaling law, and in the highly concentrated region the coil size begins to increase again. The dynamical properties, the self-diffusion coefficient of the center of mass, $D_{\mathrm{cm}}$, the relative viscosity $\eta_{r}$, and the specific viscosity $\eta_{\mathrm{sp}}$ also show the correct trends in both dilute and concentrated regions. In the highly concentrated region, however, there is a return to Rouse-like behavior-which is not realistic. This is a deficiency of the coarse-graining procedure which we suggest fails to capture important aspects of the polymer en- 
tanglement, which would require a model including physical connectivity of the beads.

We conclude that the coarse-grained segment-segment interactions capture quite well the static properties of real polymer chains in the whole concentration range. Although the contraction of polymer coils in the concentrated region in our model is more extreme than in a real polymer solution. This indicates that in the concentrated region the topological constraints or entanglements of chains present should restrict significantly the decrease of coil size with increasing concentration, which will limit the ability of the polymer molecules to contract to avoid the interchain excluded volume repulsions.

The entanglement effects influence the dynamical behavior of the chains in the concentrated region and dominate those in the highly concentrated region. In real polymers the entanglement makes $D_{\mathrm{cm}}$ decrease more dramatically with increasing concentration. It also makes $\eta_{\mathrm{sp}}$ increase faster in the concentrated region and keep increasing in the highly concentrated region. Clearly, excluded-volume aspects of the present model do not represent the real entanglement effects originating in the uncrossability of the chains, which dominates the dynamical behavior in the very concentrated state. Nevertheless, our model does have many satisfactory features, bearing in mind its simplicity and computational efficiency, and should form the basis for future refinements.

\section{ACKNOWLEDGMENTS}

C.X. thanks the Biotechnology and Biological Sciences Research Council of Great Britain (BB-SRC) for financial support and for the funding of DIGITAL AlphaStations workstations used to carry out some of these simulations. Useful discussions with Dr. R. K. Richardson and Dr. A. Haque, Department of Food Technology, Cranfield University (Silsoe Campus) are gratefully acknowledged. Some calculations were carried out on the DIGITAL computer COLUMBUS at the Rutherford Laboratory, U.K., funded by the Engineering and Physical Sciences Research Council of Great Britain (EPSRC) via a grant from the U.K. Computational Chemistry Working Party.
[1] T. G. Fox, J. B. Kingsinger, H. F. Mason, and E. M. Schuele, Polymer 3, 71 (1962).

[2] N. Kuwahara, T. Okazawa, and J. Kaneko, J. Chem. Phys. 47, 3357 (1967).

[3] Y. Miyaki, Y. Einaga, and H. Fujita, Macromolecules 11, 1180 (1978).

[4] I. Noda, N. Kato, T. Kitano, and N. Negasawa, Macromolecules 14, 668 (1981).

[5] L. Schafer, Macromolecules 15, 652 (1982).

[6] G. M. McKenna, Macromolecules 20, 498 (1987).

[7] G. Robinson, S. B. Ross-Murphy, and E. R. Morris, Carbohydrate Research 107, 17 (1982).

[8] F. M. Goycoolea, R. K. Richardson, E. R. Morris, and M. Gidley, Macromolecules 28, 8308 (1995).

[9] B. Launey, G. Cuvelier, and S. Martinez-Reyes, Carbohydrate Polym. 34, 385 (1997).

[10] P. J. Flory, J. Chem. Phys. 17, 303 (1949).

[11] J. des Cloizeaux, J. Phys. (France) 36, 281 (1975).

[12] J. C. le Guillou and J. Zinn-Justin, Phys. Rev. Lett. 39, 95 (1977).

[13] P.-G. de Gennes, Scaling Concepts in Polymer Physics (Cornell University Press, Ithaca, 1979).

[14] Y. Miyaki, Y. Einaga, T. Hirosye, and H. Fujita, Macromolecules 10, 1356 (1978).

[15] T. Hirosye, Y. Einaga, and H. Fujita, Polym. J. (Tokyo) 11, 819 (1979).

[16] G. Meyerhoff and B. Appelt, Macromolecules 12, 2103 (1979).

[17] T. Norisuye and H. Fujita, Polym. J. (Tokyo) 14, 143 (1982).

[18] A. Barret, Macromolecules 17, 1561 (1984).

[19] A. Yethiraj and R. Dickman, J. Chem. Phys. 97, 4468 (1992).

[20] P. Cifra and T. Bleha, Polymer 34, 4924 (1993).

[21] P. Cifra and T. Bleha, Macromol. Theory Simul. 4, 405 (1995).

[22] A. Milchev, W. Paul, and K. Binder, J. Chem. Phys. 99, 4786 (1993).
[23] V. Tries, W. Paul, J. Bashnagel, and K. Binder, J. Chem. Phys. 106, 738 (1997).

[24] J. Han, R. L. Jaffe, and D. Y. Yoon, Macromolecules 30, 7245 (1997).

[25] J. P. Wittmer, A. Milchev, and M. E. Cates, J. Chem. Phys. 109, 834 (1998).

[26] P. E. Rouse, J. Chem. Phys. 21, 1272 (1953).

[27] P. J. Flory, Principles of Polymer Chemistry (Cornell University Press, Ithaca, 1953).

[28] H. Yamakawa, Modern Theory of Polymer Solutions (Harper and Row, New York, 1971).

[29] H. Fujita, Polymer Solutions (Elsevier Science Publishers B.V., Amsterdam, 1990).

[30] M. Doi and S. F. Edwards, The Theory of Polymer Dynamics (Oxford University Press, Oxford, 1986).

[31] B. H. Zimm, J. Chem. Phys. 24, 269 (1956).

[32] M. Lax, Rev. Mod. Phys. 38, 541 (1966).

[33] R. Zwanzig, Adv. Chem. Phys. 15, 325 (1969).

[34] D. L. Ermak and J. A. McCammon, J. Chem. Phys. 69, 1352 (1978).

[35] H. R. Warner, Jr., Ind. Eng. Chem. Fundam. 11, 379 (1972).

[36] R. Zwanzig, Annu. Rev. Phys. Chem. 16, 67 (1965).

[37] H. Yamakawa and G. Tanaka, J. Chem. Phys. 61, 4535 (1974).

[38] W. H. Stockmayer, G. Wilemski, H. Yamakawa, and G. Tanaka, J. Chem. Phys. 63, 1039 (1975).

[39] G. D. J. Phillies, Macromolecules 28, 8198 (1995).

[40] M. Daoud, J. P. Cotton, B. Farnoux, G. Jannink, G. Serma, H. Benoit, R. Duplessix, C. Picot, and P.-G. de Gennes, Macromolecules 8, 804 (1975).

[41] W. W. Graessley, Polymer 21, 259 (1980).

[42] J. S. King, W. Boyer, G. D. Wignall, and R. Ullman, Macromolecules 18, 709 (1985).

[43] W. Brostow and M. Drewmak, J. Chem. Phys. 105, 7135 (1996).

[44] L. Leger, H. Hervet, and F. Rondelez, Macromolecules 14, 1732 (1981). 